\title{
EFFECT OF PRESSURE STRESS WORK AND VSCOUS DISSIPATION IN NATURAL CONVECTION FLOW ALONG A VERTICAL FLAT PLATE WTH HEAT CONDUCTION
}

\author{
Md. M. Alam¹, M. A. Alim² and Md. M. K. Chowdhury²
}

${ }^{1}$ Department of Mathematics, Dhaka University of Engineering and Technology, Gazipur-1700, Bangladesh.

${ }^{2}$ Department of Mathematics, Bangladesh University of Engineering and Technology, Dhaka-1000, Bangladesh.

Email: maalim@math.buet.ac.bd

\begin{abstract}
In this paper, the effect of viscous dissipation and pressure stress work on free convection flow along a vertical flat plate has been investigated. Heat conduction due to wall thickness $b$ is considered in this investigation. With a goal to attain similarity solutions of the problem posed, the developed equations are made dimensionless by using suitable transformations. The non-dimensional equations are then transformed into non-linear equations by introducing a non-similarity transformation. The resulting non-linear similar equations together with their corresponding boundary conditions based on conduction and convection are solved numerically by using the finite difference method along with Newton's linearization approximation. Numerical results for the details of the velocity profiles, temperature profiles, skin friction coefficients and the surface temperature distributions are shown both on graphs and tabular form for different values of the parameters entering into the problem.
\end{abstract}

Keywords: Free convection, viscous dissipation, pressure work and conduction.

\begin{tabular}{|c|c|c|c|}
\hline \multicolumn{4}{|c|}{ NOMENCLATURE } \\
\hline$b$ & Plate thickness & $T_{\infty}$ & Fluid asymptotic temperature \\
\hline$C_{P}$ & Specific heat & $\bar{u}, \bar{v}$ & Velocity components along $\bar{x}, \bar{y}$ directions \\
\hline$d$ & $\left(T_{b}-T_{\infty}\right) / T_{\infty}$ & $u, v$ & $\begin{array}{l}\text { respectively. } \\
\text { Dimensionless velocity components }\end{array}$ \\
\hline$f$ & Dimensionless stream function & $\bar{x}, \bar{y}$ & Cartesian coordinates \\
\hline$g$ & Acceleration due to gravity & $x, y$ & Dimensionless Cartesian coordinates \\
\hline$L$ & Reference length, $v^{2 / 3} / g^{1 / 3}$ & \multicolumn{2}{|c|}{ Greek Symbol } \\
\hline$l$ & Length of the plate & $\beta$ & Co-efficient of thermal expansion \\
\hline$N$ & Viscous dissipation parameter & $\psi$ & Stream function \\
\hline$p$ & $\begin{array}{l}\text { Coupling parameter, } \\
p=\left(k_{f} / k_{s}\right)(b / L) d^{1 / 4}\end{array}$ & $\kappa_{\mathrm{f}}, \kappa_{\mathrm{s}}$ & Fluid and solid thermal conductivities \\
\hline $\operatorname{Pr}$ & Prandtl number & $\eta$ & Dimensionless similarity variable \\
\hline$T$ & Temperature & $\rho$ & Density of the fluid \\
\hline$T_{b}$ & $\begin{array}{l}\text { Temperature at outer surface of the } \\
\text { plate }\end{array}$ & $\theta$ & Dimensionless temperature \\
\hline$T_{\mathrm{s}}$ & Solid temperature & & \\
\hline
\end{tabular}




\section{Introduction}

Free convection flow is often encountered in cooling of nuclear reactors or in the study of the structure of stars and planets. The study of temperature and heat transfer is of great importance to the engineers because of its almost universal occurrence in many branches of science and engineering. Although heat transfer analysis is most important for the proper sizing of fuel elements in the nuclear reactors cores to prevent burnout. The viscous dissipation effect plays an important role in natural convection in various devices which are subjected to large deceleration or which operate at high rotational speeds and also in strong gravitational field processes on large scales (on large planets) and in geological processes. The discussion and analysis of natural convection flows, pressure and viscous stress work effects are generally ignored but here we have considered the effects of viscous dissipation and pressure work on a natural convection flow along a vertical flat plate with heat conduction. The influence and importance of viscous stress work effects in laminar flows have been examined by Gebhart (1962) and Gebhart and Mollendorf (1969). In both of the investigations special flows over semi-infinite flat surfaces parallel to the direction of body force were considered. Gebhart (1962) considered flows generated by the plate surface temperatures, which vary as powers of $\xi$ (the distance along the plate surface from the leading edge), and Mollendorf (1969) considered flows generated by plate surface temperatures, which vary exponentially in $\xi$. Zakerullah (1972) has been investigated the viscous dissipation and pressure work effects in axisymmetric natural convection flows. Ackroyd (1974) studied the stress work effects in laminar flat plate natural convection flow. Takhar and Soundalgekar (1980) have studied the effects of viscous and Joule heating on the problem posed by Sparrow and Cess (1961), using the series expansion method of Gebhart (1962). Joshi and Gebhart (1981) have shown that the effect of pressure stress work and viscous dissipation in some natural convection flows. Pozzi and Lupo (1988) studied the coupling of conduction with laminar natural convection along a flat plate. Miyamoto et al. (1980) has been investigated the effect of axial heat conduction in a vertical flat plate on free convection heat transfer.

In the present work, we have investigated the viscous dissipation and pressure work effect on the skin friction and the surface temperature distribution in the entire region from leading edge to down stream of a viscous incompressible flow along a semi-infinite vertical flat plate. The entire thermo-fluid dynamic field resulting from the coupling of natural convection along and conduction inside the heated plate has been considered. The transformed non similar boundary layer equations governing the flow together with the boundary conditions based on conduction and convection were solved numerically using the Keller box (implicit finite difference) method, Cebeci and Bradshaw (1984) along with Newton's linearization approximation method in the entire region. We have studied the effect of the Prandtl number $P r$, the viscous dissipation parameter $N$ and pressure work parameter $\in$ on the velocity and temperature profiles as well as on the skin friction and surface temperature.

\section{Governing Equations of the Flow}

Steady two dimensional laminar free convection boundary layer flow of a viscous incompressible fluid along one side of a semi-infinite vertical flat plate of thickness ' $b$ ' insulated on the edges with temperature $T_{b}$. The flow configuration and the coordinates system are shown in Fig. 1. The mathematical statement of the basic conservation laws of mass, momentum and energy for the steady viscous incompressible flow are:

$$
\begin{aligned}
& \nabla \cdot \vec{q}=0 \\
& \rho(\vec{q} \cdot \nabla) \vec{q}=-\nabla P+\mu \nabla^{2} \vec{q}+\vec{F} \\
& \rho C_{p}(\vec{q} \cdot \nabla) T-(\vec{q} \cdot \nabla) P=\nabla \cdot(\kappa \nabla T)+\mu \phi
\end{aligned}
$$

Where $\vec{q}=(\bar{u}, \bar{v}), \bar{u}$ and $\bar{v}$ are the velocity components along the $\bar{x}$ and $\bar{y}$ - axes respectively, $\vec{F}$ is the body force per unit volume which is defined as $-\rho g, T$ is the temperature of the fluid in the boundary layer, $g$ is the acceleration due to gravity, $\kappa$ is the thermal conductivity and $C_{P}$ is the specific heat at constant pressure and $\mu$ is the viscosity of the fluid. In the energy equation the viscous dissipation and pressure work terms are included. After introducing Boussinesq approximation, $\rho=\rho_{\infty}\left[1-\beta\left(T-T_{\infty}\right)\right]$ the basic equations (1) to (3) become:

$$
\frac{\partial \bar{u}}{\partial \bar{x}}+\frac{\partial \bar{v}}{\partial \bar{y}}=0
$$




$$
\bar{u} \frac{\partial \bar{u}}{\partial \bar{x}}+\bar{v} \frac{\partial \bar{u}}{\partial \bar{y}}=v \frac{\partial^{2} \bar{u}}{\partial \bar{y}^{2}}+g \beta\left(T-T_{\infty}\right)
$$

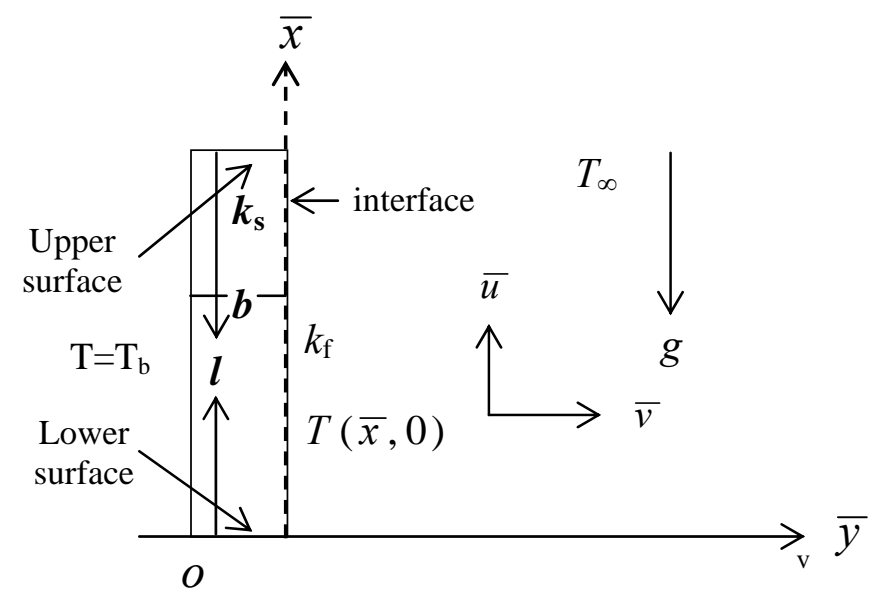

Fig.1: Physical configuration and coordinates system

$$
\bar{u} \frac{\partial T}{\partial \bar{x}}+\bar{v} \frac{\partial T}{\partial \bar{y}}=\frac{\kappa}{\rho c_{p}} \frac{\partial^{2} T}{\partial \bar{y}^{2}}+\frac{v}{c_{p}}\left(\frac{\partial \bar{u}}{\partial \bar{y}}\right)^{2}+\frac{T \beta \bar{u}}{\rho C_{p}} \frac{\partial P}{\partial \bar{x}}
$$

The appropriate boundary conditions to be satisfied by the above equations are

$$
\begin{aligned}
& \bar{u}=\bar{v}=0 \text { at } \bar{y}=0 \\
& \bar{u} \rightarrow 0, T \rightarrow T_{\infty} \text { as } \bar{y} \rightarrow \infty
\end{aligned}
$$

The temperature and the heat flux are considered continuous at the interface for the coupled conditions and at the interface we must have

$$
\frac{k_{s}}{k_{f}} \frac{\partial T_{s o}}{\partial \bar{y}}=\left(\frac{\partial T}{\partial \bar{y}}\right)_{\bar{y}=0}
$$

Where $k_{s}$ and $k_{f}$ are the thermal conductivity of the solid and the fluid respectively. The temperature $T_{s o}$ in the solid as given by Pozzi and Lupo (1988) is

$$
T_{s o}=T(\bar{x}, 0)-\left\{T_{b}-T(\bar{x}, 0)\right\} \frac{\bar{y}}{b}
$$

Where $T(\bar{x}, 0)$ is the unknown temperature at the interface to be determined from the solutions of the equations. We observe that the equations (4) - (6) together with the boundary conditions (8) - (9) are non-linear partial differential equations, which have been solved numerically and are described in the following sections.

\section{Transformation of the Governing Equations}

Equations (4)-(6) may now be non-dimensionalized by using the following dimensionless dependent and independent variables:

$$
x=\frac{\bar{x}}{L}, y=\frac{\bar{y}}{L} d^{1 / 4}, \bar{u}=\frac{v}{L} d^{1 / 4} u, \bar{v}=\frac{v}{L} d^{1 / 4} v, \theta=\frac{T-T_{\infty}}{T_{b}-T_{\infty}}, L=\frac{v^{2 / 3}}{g^{1 / 3}}, d=\beta\left(T_{b}-T_{\infty}\right)
$$

As this natural convection problem is of parabolic nature the characteristic length, $L$ has been defined in terms of $v$ and $g$, which are the intrinsic properties of the system. The reference length along the ' $y$ ' direction has been modified by a factor $\mathrm{d}^{-1 / 4}$ in order to eliminate this quantity from the dimensionless equations and the boundary conditions.

Using the above relations (10) the non-dimensional form of the governing equations are: 


$$
\begin{aligned}
& \frac{\partial u}{\partial x}+\frac{\partial v}{\partial y}=0 \\
& u \frac{\partial u}{\partial x}+v \frac{\partial u}{\partial y}=\frac{\partial^{2} u}{\partial y^{2}}+\theta \\
& u \frac{\partial \theta}{\partial x}+v \frac{\partial \theta}{\partial y}=\frac{1}{\operatorname{Pr}} \frac{\partial^{2} \theta}{\partial y^{2}}+N\left(\frac{\partial u}{\partial y}\right)^{2}-\frac{g \beta\left\{T_{\infty}+\left(T_{b}-T_{\infty}\right) \theta\right\}}{C_{p}\left(T_{b}-T_{\infty}\right)}
\end{aligned}
$$

Where $P_{r}=\mu C_{p} / \kappa_{f}$ is the Prandtl number and $N=v^{2} d / L^{2} C_{p}\left(T_{b}-T_{\infty}\right)$, the dimensionless viscous dissipation parameter. For exterior conditions, we know hydrostatic pressure, $\partial P / \partial x=\rho_{e} g$ and $\rho=\rho_{e}$, and the pressure work parameter $\in=g \beta x / C_{p}$ which is less than one as suggested by Gebhart (1962).

The corresponding boundary conditions (7) - (8) take the following form:

$$
\begin{aligned}
& u=v=0, \theta-1=p \frac{\partial \theta}{\partial y} \text { at } y=0 \\
& u \rightarrow 0 v \rightarrow 0 \text { as } y \rightarrow \infty
\end{aligned}
$$

where $P$ is the pressure and $p$ is the conjugate conduction parameter given by $p=\left(\kappa_{f} / \kappa_{\mathrm{s}}\right)(b / L) \mathrm{d}^{1 / 4}$. Here the coupling parameter ' $p$ ' governs the described problem. The order of magnitude of ' $p$ ' depends actually on $b / L$ and $\kappa_{f} /$ $\kappa_{s}, d^{1 / 4}$ being the order of unity. The term $b / L$ attains values much greater than one because of $L$ being small. In case of air, $\kappa_{f} / \kappa_{\mathrm{s}}$ becomes very small when the vertical plate is highly conductive i.e. $\kappa_{\mathrm{s}}>>1$ and for materials, $\mathrm{O}\left(\kappa_{f}\right)$ $\left.\kappa_{\mathrm{s}}\right)=0.1$ such as glass. Therefore in different cases ' $p$ ' is different but not always a small number. In the present investigation we have considered $p=1$ which is accepted for $b / L$ of $\mathrm{O}\left(\kappa_{f} / \kappa_{\mathrm{s}}\right)$. To solve the equations (12) - (13) subject to the boundary conditions (14) to (15), the following transformations were introduced for the flow region starting from up stream to down stream.

$$
\psi=x^{4 / 5}(1+x)^{-1 / 20} f(\eta, x), \quad \eta=y x^{-1 / 5}(1+x)^{-1 / 20}, \theta=x^{1 / 5}(1+x)^{-1 / 5} h(\eta, x)
$$

Here $\eta$ is the dimensionless similarity variable and $\psi$ is the stream function which satisfies the equation of continuity and $u=\frac{\partial \psi}{\partial y}, v=-\frac{\partial \psi}{\partial x}$ and $h(\eta, x)$ is the dimensionless temperature. Then the equations (12) and (13) transformed to the following non dimensional forms:

$$
\begin{aligned}
& f^{\prime \prime \prime}+\frac{16+15 x}{20(1+x)} f f^{\prime \prime}-\frac{6+5 x}{10(1+x)} f^{\prime 2}+h=x\left(f^{\prime} \frac{\partial f^{\prime}}{\partial x}-f^{\prime \prime} \frac{\partial f}{\partial x}\right) \\
& \frac{1}{\operatorname{Pr}} h^{\prime \prime}+\frac{16+15 x}{20(1=x)} f h^{\prime}-\frac{1}{5(1+x)} f^{\prime} h+N x f^{\prime \prime 2} \\
& -\in\left\{\left(\frac{1+x}{x}\right)^{1 / 5}\left(\frac{T_{\infty}}{T_{b}-T_{\infty}}\right) f^{\prime}+h f^{\prime}\right\}=x\left(f^{\prime} \frac{\partial h}{\partial x}-h^{\prime} \frac{\partial f}{\partial x}\right)
\end{aligned}
$$

In the above equations the primes denote differentiation with respect to $\eta$. The boundary conditions (14)-(15), take the following form

$$
\begin{aligned}
& f(x, 0)=f^{\prime}(x, 0)=0, h^{\prime}(x, 0)=-(1+x)^{1 / 4}+x^{1 / 5}(1+x)^{1 / 20} h(x, 0) \\
& f^{\prime}(x, \infty)=0, h^{\prime}(x, \infty)=0
\end{aligned}
$$

\section{Method of Solution}

The numerical methods used is finite difference method together with Keller box Scheme which is described in details by Keller and Cebeci (1971), Cebecci and Bradshow (1984) and widely used by Hossain and Alim (1997) and Hossain et al. (1998). 


\section{Results and Discussion}

The viscous dissipation and pressure work effect on the skin friction and the surface temperature distribution of a viscous incompressible flow along a semi-infinite vertical flat plate has been investigated and the coupling of natural convection along and conduction inside the heated plate has been considered. Solutions are obtained for the values of Prandtl number $P r=0.05,0.72,1.0,1.74$ and for a range of values of the viscous dissipation parameter $N=0.1,0.3$, $0.5,0.9$ and the pressure work parameter $\in=0.1,0.4,0.7,0.9$. If we know the values of the functions $f(\eta, x), h(\eta, x)$ and their derivatives for different values of the pertinent parameters then it is possible to calculate the numerical values of the surface temperature $\theta(0, x)$ and the velocity gradient (skin frictions) $f^{\prime \prime}(0, x)$ at the surface that are important from the physical point of view.
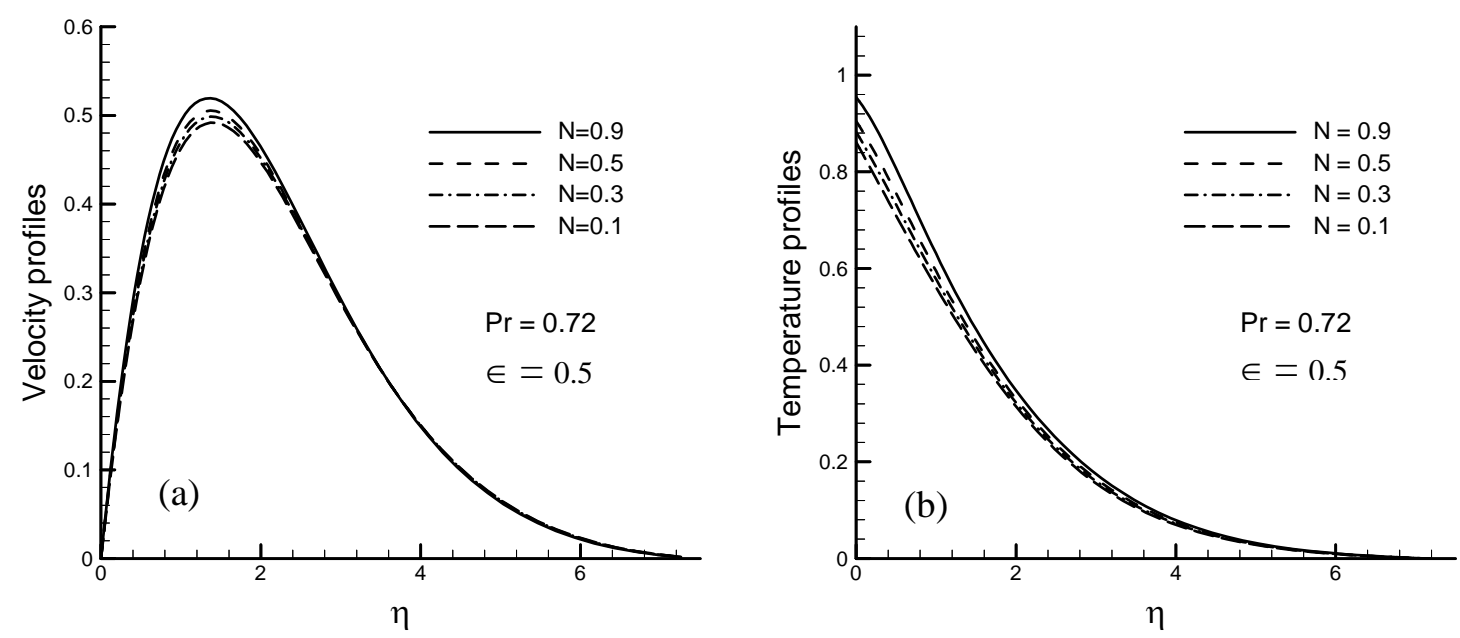

Fig.2: (a) Velocity and (b) Temperature profiles are shown against $\eta$ for different values of viscous dissipation parameter $N$ for controlling parameter $P r=0.72, \in=0.5$.

Fig.2 (a) and Fig.2 (b) show the effects of the viscous dissipation parameter $N(=0.1,0.3,0.5,0.9)$ on the velocity and the temperature profiles for $P r=0.72$ and $\in=0.5$. From Fig. 2(a), it is revealed that the velocity profile $f^{\prime}(\eta, x)$ increases with the increase of the viscous dissipation parameter $N$ which indicates that viscous dissipation increases the fluid motion slightly. In Fig. 2(b) the similar behavior has also been observed for the temperature profiles $h(\eta, x)$ with the similar values of controlling parameters $\mathrm{N}, \operatorname{Pr}$ and $\in$.

We observe from Fig. 3(a), that an increase in the pressure work parameter, $\epsilon$, is increases the velocity profiles but near the surface of the plate the velocity increases and become maximum and then decreases and finally approaches to zero. The maximum values of the velocities are $0.5129,0.5228,0.5352,0.5542$ for $\in=0.1,0.4,0.7,0.9$ respectively and each of which occurs at $\eta=1.3025$ for the first maximum value and $\eta=1.3693$ for the $2^{\text {nd }}, 3^{\text {rd }}$, fourth maximum values. Here we found that the velocity increases by $8.0523 \%$ as $\in$ increases from 0.1 to 0.9 . However Fig. 3(b) shows the distribution of the temperature profiles $h(\eta, x)$ against $\eta$ for some values of the pressure work parameter $\in(=0.1,0.4,0.7,0.9)$. Clearly it is seen that the temperature distribution increases owing to increasing values of the pressure work parameter $\in$ and becomes maximum at the wall. The local maximum values of the temperature profiles are $0.8962,0.9283,0.9641,0.9937$ for $\in(=0.1,0.4,0.7,0.9)$ respectively and each of which attains at the surface. Thus the temperature profiles increase by $10.8793 \%$ as $\in$ increases from 0.1 to 0.9 .

Figs. 4 (a) and (b) illustrates the velocity and temperature profiles for different values of Prandtl number in presence of viscous dissipation and pressure work terms. From Fig. 4 (a), we may conclude that the velocity profile decreases when the value of the Prandtl number $P r$ increases. But it is seen that near the surface of the flat plate the velocity increases considerably and become maximum and then decreases slowly and finally approaches to zero. The maximum values of the velocities are $0.3679,0.4476,0.4987,0.9749$ for $\operatorname{Pr}=1.74,1.0,0.77,0.05$ respectively which occur at $\eta=1.3025$ for the first maximum value, $\eta=1.3693$ for the second and third maximum values and at $\eta=1.6593$ for the last maximum value. Here it is found that the velocity profiles decrease by $62.263 \%$ while $\mathrm{Pr}$ increases from 0.05 to 1.74. On the other hand, in the case of temperature field, from Fig. 4(b), we observed that the temperature distribution over the whole boundary layer decreases significantly when the values of the Prandtl 
number $P r$ increases. Here we observed that the maximum values of the temperature profiles are 1.0555, 0.8522, 0.8271, 0.7844 for $P r=0.05,0.7,1.0,1.74$ respectively and each of which attains at the surface of the plate. Thus in this case temperature profiles decrease by $28.684 \%$
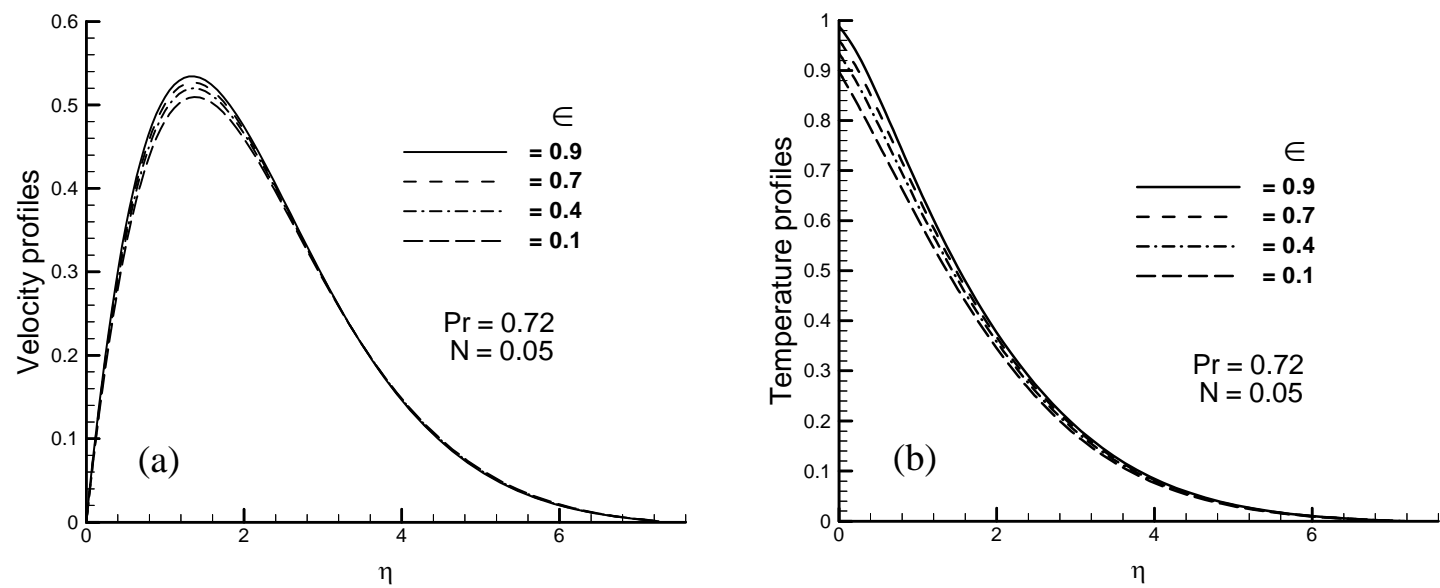

Fig.3: (a) Velocity and (b) Temperature profiles are shown against $\eta$ for different values of pressure work parameter $\in$ for controlling parameter $\operatorname{Pr}=0.72, N=0.05$.
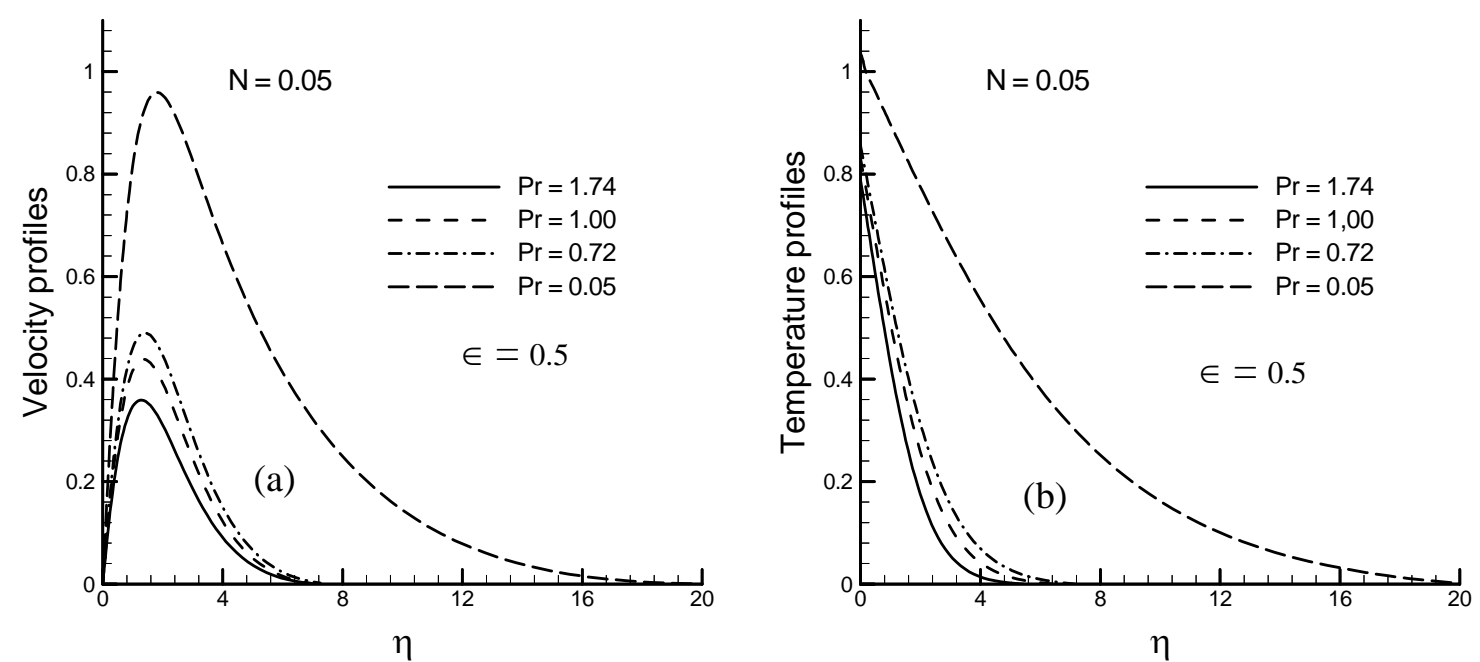

Fig. 4: (a) Velocity and (b) Temperature profiles are shown against $\eta$ for different values of Prandtl number $\operatorname{Pr}$ for controlling parameter $N=0.05, \in=0.5$.

Numerical values of the velocity gradient $f^{\prime \prime}(0, x)$ and the surface temperature $\theta(0, x)$ are depicted graphically in Fig.5 (a) and 5(b) respectively against the axial distance $x$ for different values of the viscous dissipation parameter $N$ (=0.1, 0.3, 0.5, 0.9) for the fluid having Prandtl number $\operatorname{Pr}=0.72$ and pressure work parameter $\in=0.5$. From Fig. 5 (a), it can be seen that an increase in the dissipative heat is associated with the enhancing of the skin friction coefficient. Same result is seen in Fig. 5 (b) for surface temperature distribution.

In Fig.6 (a), the shear stress coefficient $f^{\prime}(0, x)$ and Fig.6 (b), the surface temperature distribution $\theta(0, x)$ are shown graphically for different values of the Prandtl number $\operatorname{Pr}(=0.05,0.72,1.0,1.74)$ with fixed parameter $N=0.05$ and $\in=0.5$. Four values 0.05 (mercury), 0.72 (air), 1.0 (salt water), 1.74 (theoretically) are taken for $P r$, the Prandtl number. From the first figure, 6(a) it can be seen that for the increased values of Prandtl number, $P r$ decrease the shear stress coefficient and the same result is observed from Fig. 6(b) for surface temperature distribution. 
In Fig.6 (a), the shear stress coefficient $f^{\prime \prime}(0, x)$ and Fig.6 (b), the surface temperature distribution $\theta(0, x)$ are shown graphically for different values of the Prandtl number $\operatorname{Pr}(=0.05,0.72,1.0,1.74)$ with fixed parameter $N=0.05$ and $\in=0.5$. Four values 0.05 (mercury), 0.72 (air), 1.0 (salt water), 1.74 (theoretically) are taken for $P r$, the Prandtl number. From the first figure, 6(a) it can be seen that for the increased values of Prandtl number, $\operatorname{Pr}$ decrease the shear stress coefficient and the same result is observed from Fig. 6(b) for surface temperature distribution.
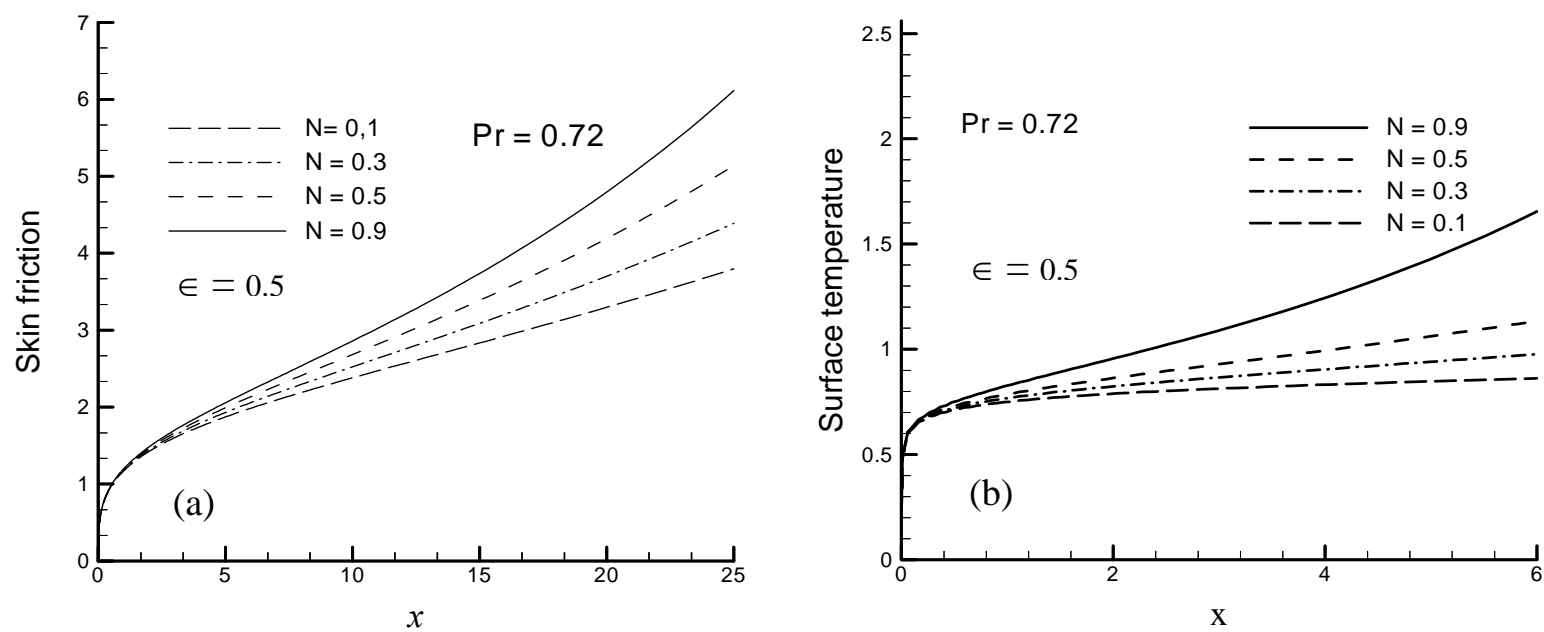

Fig.5: (a) Shear stress and (b) Surface temperature are depicted against $x$ for different values of viscous dissipation $N$ with others fixed controlling parameters $\operatorname{Pr}$ and $\in$.
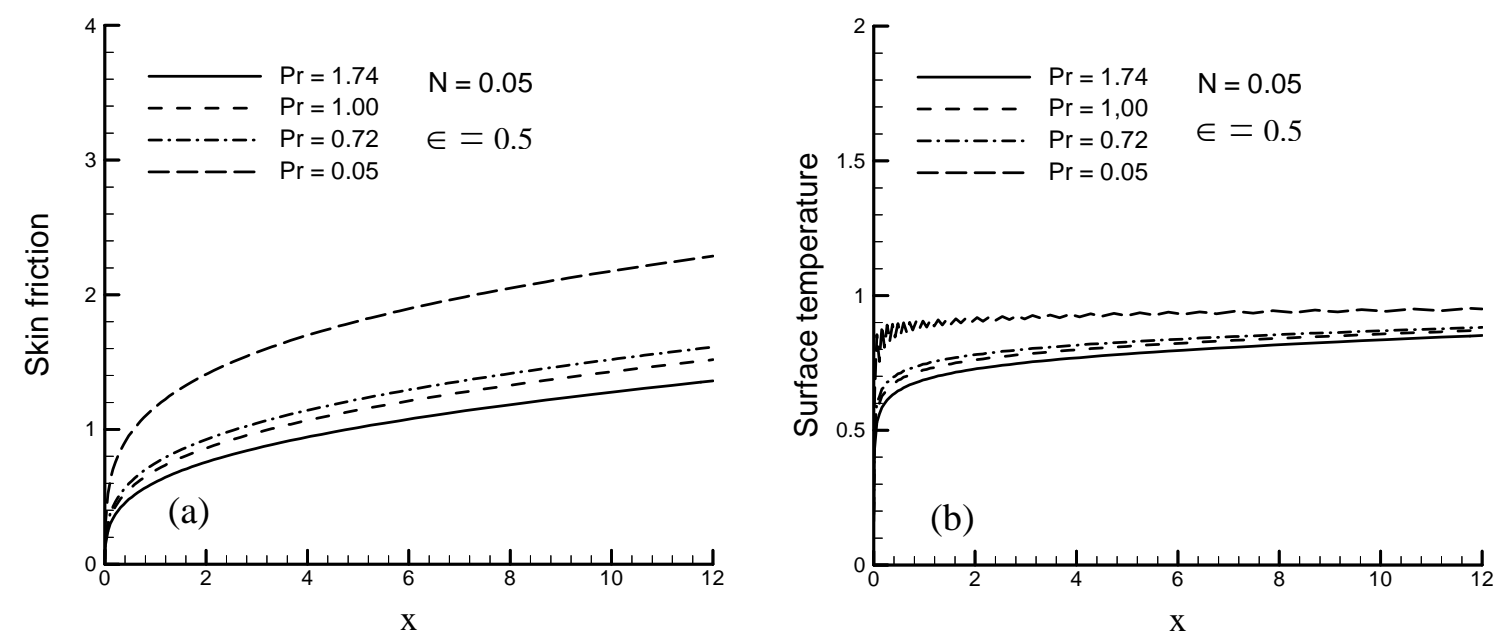

Fig. 6: (a) Shear stress and (b) Surface temperature are depicted against $x$ for different values of Prandtl number $\operatorname{Pr}$ with others controoling parameters $N$ and $\in$.

\section{Conclusions}

The effect of viscous dissipation and the pressure work on natural convection boundary layer flow from a vertical flat plate with heat conduction inside the heated plate has been studied. The transformed non-similar boundary layer equations governing the flow together with the boundary conditions based on conduction and convection were solved numerically using the implicit finite difference method together with Keller box scheme. The coupled effect of natural convection and conduction required that the temperature and the heat flux be continuous at the interface. The numerical values of skin friction, the surface temperature distribution, velocity and temperature profiles have been presented graphically and in tabular form. From the present investigation, the following conclusions may be drawn: 
- The skin friction, the velocity and the temperature distributions increase for increasing value of the viscous dissipation parameter $N$.

- The surface temperature, the velocity and the temperature distributions increase for increasing values of the pressure work parameter $\epsilon$.

- It has been observed the skin friction, the temperature distribution over the whole boundary layer and the velocity distribution decrease with the increase of the Prandtl number $P r$.

Table-1: Skin friction coefficient and surface temperature against $x$ for different values of pressure work parameter $\in$ with other controlling parameters $\operatorname{Pr}=0.72, N=0.05$.

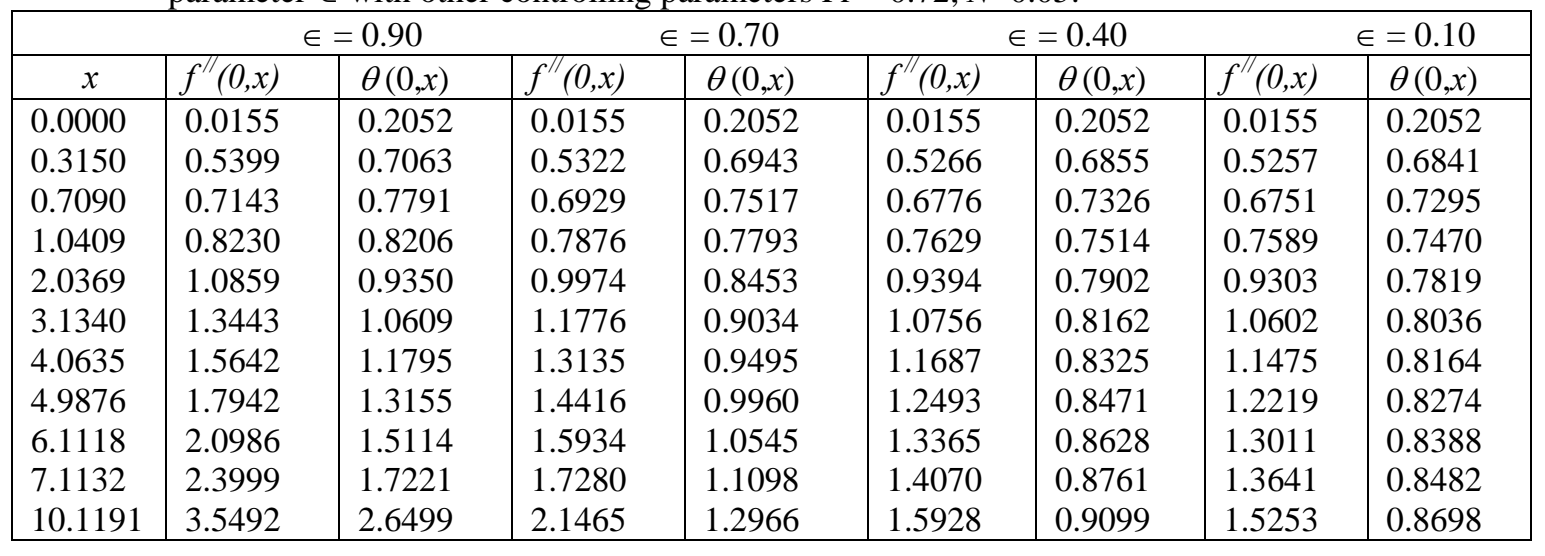

\section{References}

Ackroyd, J. A. D. (1974): Stress Work Effects in Laminar Flat-plate Natural Convection, J. Fluid Mech., Vol. 62, pp. 677-695.

Cebeci, T. and Bradshaw, P. (1984): Physical and Computational Aspects of Convective Heat Transfer, Springer, New York.

Gebhart, B. (1962): Effects of Viscous Dissipation in Natural Convection, J. Fluid Mech., Vol. 14, pp. 225-232.

Gebhart, B., and Mollendorf, J. (1969): Viscous Dissipation in External Natural Convection Flows. J. Fluid Mech, Vol.38, pp. 97-107.

Hossain, M. A. and Alim, M. A. (1997): Natural Convection-radiation Interaction on Boundary Layer Flow along a Thin Cylinder, J. Heat and Mass Transfer Vol. 32, pp.515-520.

Hossain, M. A., Alim, M. A., and Rees, D.A.S. (1998): Effects of Thermal Radiation on Natural Convection over Cylinders of Elliptic Cross Section. Acta Mechanica, Vol. 129, pp. 177-186.

Joshi,Y. and Gebhart, B. (1981): Effect of pressure stress work and viscous dissipation in some natural convection flows, Int. J. Heat Mass Transfer, Vol. 24, No. 10, pp. 1377-1388.

Keller, H. B., \& Cebeci, T. (1971): Accurate Numerical Methods for Boundary Layer Flows, part-I. TwoDimensional Laminar Flows”, Proceedings of the Second International Conference on numerical Methods in fluid dynamics, p. 92. Springer, New York.

Miyamoto, M., Sumikawa, J., Akiyoshi, T. and Takamura, T. (1980): The Effect of Axial Heat Conduction in a Vertical Flat Plate on Free Convection Heat Transfer. Int. J. Heat Mass Transfer, V. 23, pp. 1545-1553.

Pozzi, A. and Lupo, M. (1988): The Coupling of Conduction with Laminar Natural Convection along a Flat Plate, Int. J. Heat Mass Transfer. Vol. 31, No. 9, pp. 1807-1814.

Sparrow, E.M. \& Cess, R. D. (1961): The Effect of Magnetic Field on Free Convention Heat Transfer, Int. J. Heat and Mass Transfer, Vol. 3, pp. 267.

Takhar, H. S. and Soundalgekar, V. M. (1980): Dissipation Effects on MHD Free Convection Flow Past a semiinfinite Vertical Plate, Applied Scientific Research. Vol. 36 pp.163-171.

Zakerullah, M. (1972): Viscous Dissipation and Pressure Work Effects in Axisymmetric Natural Convection Flows, Ganit(J. Bangladesh Math. Soc.) Vol. 2. No.1, pp. 43. 